\title{
Modified Radius Directed Equaliser for High Order QAM
}

\author{
Domaniç Lavery*, Milen Paskov, Robert Maher, Seb J. Savory and Polina Bayvel
}

Optical Networks Group, Department of Electronic \& Electrical Engineering, University College London, Torrington Place, London WC1E 7JE, United Kingdom. *d.lavery@ee.ucl.ac.uk

\begin{abstract}
The RDE for M-ary QAM equalisation is modified using a low-complexity probabilistic update algorithm. Performance is investigated in simulation and experiment for 8 GBd DP-64QAM and DP256QAM. DP-64QAM improved by $3.2 d B Q^{2}$-factor for a dynamic channel with high OSNR.
\end{abstract}

\section{Introduction}

Recent demonstrations of high capacity optical communications links have made use of high order quadrature amplitude modulation (QAM) in order to increase spectral efficiency ${ }^{1}$. In particular, dual polarisation (DP)-32-, 64- and 256-ary QAM.

Whilst it is relatively straightforward to equalise the time invariant channel using fixed tap weight finite impulse response (FIR) filters (e.g., for digital chromatic dispersion compensation, matched filtering), the dynamic channel (e.g., differential group delay, polarisation rotations) requires adaptive equalisation. The conventional approach for DP quadrature phase shift keying (QPSK) has been to use the constant modulus algorithm (CMA) to update adaptive FIR filters in a $2 \times 2$ multiple input multiple output (MIMO) structure, which tracks the state of polarisation, recovers the sampling instant, and compensates for any residual static channel distortions ${ }^{2}$.

This approach has been extended to M-ary QAM, where the CMA is used to pre-converge the FIR filter tap weights before switching to a radius directed equaliser (RDE), which makes decisions on the modulus of the symbol before updating the tap weights ${ }^{3}$. Note this is equivalent to the multimodulus algorithm (MMA). This blind equalisation approach for 32- and 64QAM has been experimentally verified ${ }^{1,4}$.

The disadvantage of the blind algorithms is that they are not robust to fast changes in the transmission channel, and this tracking ability is impaired as the order of QAM is increased.

We note that, whilst it is also possible to equalise the signal based on training or pilot symbols, this reduces the maximum achievable spectral efficiency, and requires frame synchronisation before equalisation, limiting acquisition time.

In this paper, we propose a simple modification to the RDE which enhances the tracking ability of this equaliser, with a computational complexity of just two additional real-valued multiplications (one per polarisation) per iteration.

\section{Modified Radius Directed Equaliser}

The blind algorithms outlined above are from the class of equalisers known as property restoral filters; in this case, restoring the property of a known (finite) number of radii. We observe that an additional property of the signal is the relative probability of the radii, based on the number of constellation points in each radius. The error term for these equalisers can then be expressed as

$$
\varepsilon_{x}(k)=P(r)\left(r^{2}-\left|x_{\text {out }}(k)\right|^{2}\right)
$$

where $k$ is the sampling instant, $x_{\text {out }}(k)$ the output symbol on polarisation $X, r$ is the hard decision on the radius of $x_{\text {out }}(k)$, and $P(r)$ is the relative probability of receiving a radius $r$ (given the order of QAM transmitted). The error can be computed similarly for the Y-polarisation. Note that the error term of the probabilistic RDE (PRDE), defined in Eq. (1), becomes the conventional $\mathrm{RDE}^{1}$ by setting $P(r)=1$. The equaliser filter tap weight updates are computed as in Ref. ${ }^{3}$.

\section{Simulation and Experiment}

The performance of the RDE and the PRDE algorithms were first evaluated via numerical simulations. DP-64QAM and DP-256QAM signals were generated with randomly chosen symbol sequences of length $2^{18}$. The signal was sampled at 4 samples per symbol before applying a root raised cosine (RRC) filter (rolloff 0.01). The channel was modelled using continuous polarisation rotations at a fixed frequency ${ }^{5}$.

The receiver was modelled as an ideal phaseand polarisation-diverse coherent receiver, followed by ideal analogue-to-digital conversion at

\footnotetext{
${ }^{1}$ To directly compare PRDE and RDE, the average over all $P(r)$ must be unity; the approach taken here. E.g., 16QAM radii probabilities are $P(r)=(0.25,0.5,0.25)$, so the unit mean values are $P(r)=(0.75,1.5,0.75)$.
} 
two samples per symbol. The receiver DSP consisted of a matched RRC filter, followed by a 5-tap (T/2-spaced) CMA equaliser (for tap weight preconvergence) and the RDE equaliser under test. The equaliser was permitted 5 complex-valued taps in order to recover both the state of polarisation and the ideal sampling instant. The learning parameter, $\mu$, was optimised for both equalisers by selecting the optimum $\mu$ from 25 logarithmically spaced test values between $10^{-5}$ and $10^{-1}$ for each channel and each OSNR.

The experimental configuration is shown in Fig. 1(a). Waveforms were generated, as in simulation, at four samples per symbol, but uploaded to a pair of digital-to-analogue converters (DAC) (5 effective number of bits (ENOB), $16 \mathrm{GHz}$ electrical bandwidth), and output at $32 \mathrm{GSa} / \mathrm{s}$ (8 GBd). These signals were used to drive an 'IQ'-modulator, which was seeded with the output from an external cavity laser (ECL) $(1550 \mathrm{~nm}$ wavelength, $1.1 \mathrm{kHz}$ linewidth).

The modulated signal was passed to a polarisation multiplexing emulation stage (389 symbols $\mathrm{X} / \mathrm{Y}$ delay) before being received using a phaseand polarisation-diverse coherent receiver. The local oscillator laser used was an ECL (1550 nm wavelength, $1.5 \mathrm{kHz}$ linewidth). The received signal was digitised using a digital storage oscilloscope and resampled offline to $2 \mathrm{Sa} / \mathrm{symbol}$. The signal was normalised and timing skew within the coherent receiver was compensated.

At this stage, where polarisation rotations were considered, they were added digitally, as in simulations. Again, as in the simulations, $2^{18}$ symbols were processed, although here using the DSP chain shown in 1 (b). The experimental work used 51 T/2-spaced equaliser taps with a Gaussian update window, which was found to be optimum for the transmitter and receiver used. The learning parameter was optimised, following the above procedure. (Further details of the DSP can be found in Ref. ${ }^{6}$.)

\section{Results}

The simulation results for DP-64QAM and DP256QAM are shown in Figs. 2(a) and (b), respectively. Negligible performance difference between PRDE and RDE is observed in the static channel scenario (polarisation rotation rate $0 \mathrm{rad} / \mathrm{s} / \mathrm{Hz}$ ). This is to be expected as, given a sufficiently long convergence time and small learning parameter, $\mu$, the tap weights would converge to the ideal timing recovery filter. For the dynamic channel,
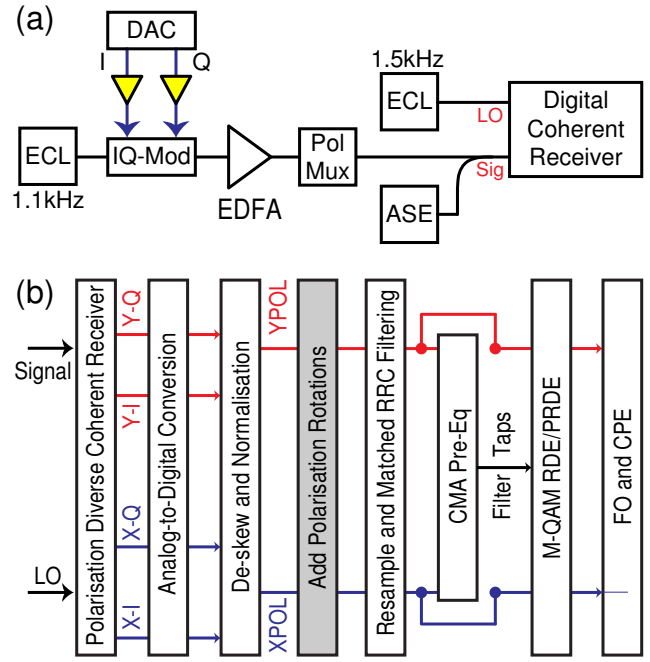

Fig. 1: (a) Experimental configuration (b) DSP chain including block used to emulate a dynamic channel (polarisation rotations).
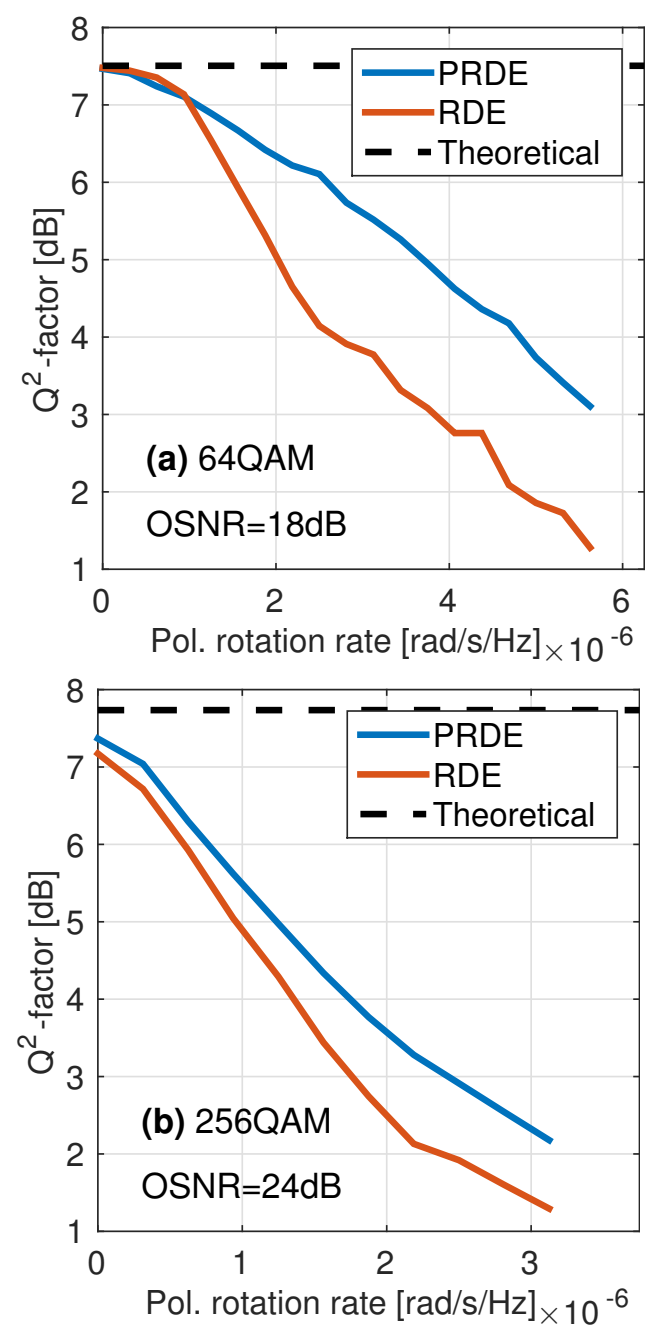

Fig. 2: Simulation results for (a) DP-64QAM, and (b) DP-256QAM. The reported $\mathrm{Q}^{2}$-factor is calculated from the raw bit error ratio. Note that, although the simulations are symbol rate independent, the OSNR is quoted assuming a symbol rate of $8 \mathrm{GBd}$. 

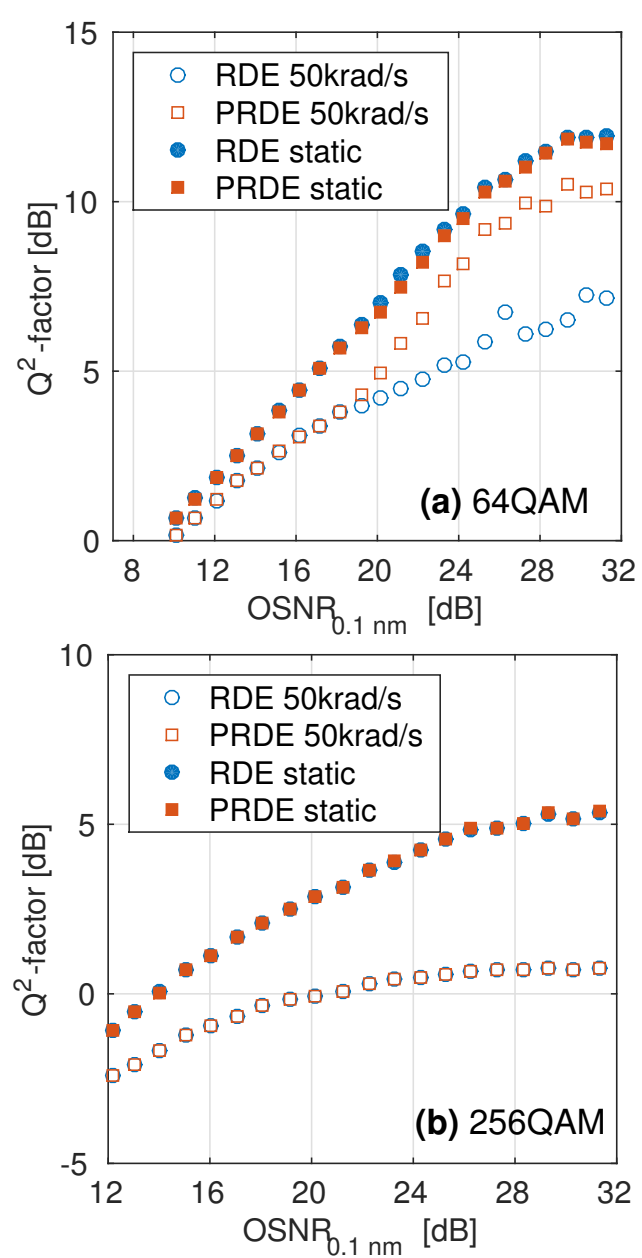

Fig. 3: Experimental results for (a) DP-64QAM, and (b) DP-256QAM.

the PRDE outperforms the RDE for all polarisation rotation rates tested and for all OSNR values tested. (Note that, although the PRDE can be applied to 16QAM, we observed no performance advantage versus the RDE for this modulation format.) For the subset of the results at a fixed OSNR in Fig. 2, observe that the $Q^{2}$-factor gain for the PRDE broadly increases with polarisation rotation rate.

For the experimental data, Fig. 3, a similar trend was observed, with the two equalisation algorithms performing similarly for the notionally static channel. When polarisation rotations were applied to the signal, it was found that, again, the PRDE outperformed the RDE. The high OSNR performance improvement was approximately $3.2 \mathrm{~dB}$ in $\mathrm{Q}^{2}$-factor (32 dB OSNR) for DP-64QAM, Fig. 3(a).

Observe that, for particularly low OSNR values, the performance of the PRDE and RDE converges. In this regime, we believe that the advantage of probabilistically updating the equaliser tap weights is outweighed by the additional uncertainty introduced by additive white noise.

For DP-256QAM, Fig. 3(b), no gain is observed for the PRDE experimentally. By the same token, it is likely that the relatively high error floor of the static channel masks any gain from the probabilistic equaliser that was observed in simulation. The simulation results indicate that, if this experimental issue were to be overcome, a gain would be also observed from the PRDE in this scenario.

\section{Conclusions}

An equalisation algorithm for DP-QAM was presented, based on the RDE, which probabilistically updates the filter tap weights based on the relative likelihood of the radii decision. This algorithm offers a significant improvement in tracking fast changes in dynamic channels, but exhibits negligible performance difference versus the RDE for a static channel. Given the low complexity of the algorithm, it may be prudent to implement this modification in scenarios where the blind RDE is being used for high order ( $\geq 64$-ary) DP-QAM.

The equalisation algorithms were investigated in this work using symbol-by-symbol tap weight updates. Future work will consider the implications of a hardware implementation on the performance of these equalisation algorithms.

\section{Acknowledgements}

The authors acknowledge EPSRC UNLOC $(\mathrm{EP} / \mathrm{J} 017582 / 1)$ and EPSRC COSINE (EP/I012702/1) projects, and The Royal Academy of Engineering / The Leverhulme Trust for financial support.

\section{References}

[1] R. Rios-Müller et al., "1-Terabit/s Net Data-Rate Transceiver Based on Single-Carrier Nyquist-Shaped 124 GBaud PDM-32QAM," Proc. OFC, Th5B.1, (2015).

[2] S. Savory, "Digital filters for coherent optical receivers," Opt. Express Vol. 16, p. 804 (2008).

[3] I. Fatadin et al., "Blind Equalization and Carrier Phase Recovery in a 16-QAM Optical Coherent System," J. Lightwave Technol., Vol. 27, no. 15, p. 3042 (2009).

[4] F. P. Guiomar, et al., "Fully Blind Linear and Nonlinear Equalization for 100G PM-64QAM Optical Systems," J. Lightwave Technol., Vol. 33, no. 7, p. 1265, (2015).

[5] D. Lavery et al., "Reduced complexity equalization for coherent long-reach passive optical networks [invited]," J. Opt. Commun. Netw., Vol. 7, no. 1, p. A16, (2015).

[6] R. Maher et al., "Spectrally Shaped DP-16QAM SuperChannel Transmission with Multi-Channel Digital Back Propagation," Scientific Reports Vol. 5, p. 8214 (2015). 